\title{
Rare deleterious mutations of the gene EFR3A in autism spectrum disorders
}

\author{
Abha R Gupta1*, Michelle Pirruccello², Feng Cheng ${ }^{3,4}$, Hyo Jung Kang ${ }^{3,5}$, Thomas V Fernandez ${ }^{6}$, Jeremy M Baskin, \\ Murim Choi ${ }^{8}$, Li Liu ${ }^{9}$, Adife Gulhan Ercan-Sencicek ${ }^{10}$, John D Murdoch ${ }^{11}$, Lambertus Klei ${ }^{12}$, Benjamin M Neale ${ }^{13}$, \\ Daniel Franjic ${ }^{3}$, Mark J Daly ${ }^{13}$, Richard P Lifton ${ }^{8}$, Pietro De Camilli ${ }^{7}$, Hongyu Zhao ${ }^{14}$, Nenad Šestan ${ }^{3}$ \\ and Matthew W State ${ }^{15^{*}}$
}

\begin{abstract}
Background: Whole-exome sequencing studies in autism spectrum disorder (ASD) have identified de novo mutations in novel candidate genes, including the synaptic gene Eighty-five Requiring $3 A$ (EFR3A). EFR3A is a critical component of a protein complex required for the synthesis of the phosphoinositide Ptdlns4P, which has a variety of functions at the neural synapse. We hypothesized that deleterious mutations in EFR3A would be significantly associated with ASD.

Methods: We conducted a large case/control association study by deep resequencing and analysis of whole-exome data for coding and splice site variants in EFR3A. We determined the potential impact of these variants on protein structure and function by a variety of conservation measures and analysis of the Saccharomyces cerevisiae Efr3 crystal structure. We also analyzed the expression pattern of EFR3A in human brain tissue.

Results: Rare nonsynonymous mutations in EFR3A were more common among cases $(16 / 2,196=0.73 \%)$ than matched controls $(12 / 3,389=0.35 \%)$ and were statistically more common at conserved nucleotides based on an experiment-wide significance threshold ( $P=0.0077$, permutation test). Crystal structure analysis revealed that mutations likely to be deleterious were also statistically more common in cases than controls ( $P=0.017$, Fisher exact test). Furthermore, EFR3A is expressed in cortical neurons, including pyramidal neurons, during human fetal brain development in a pattern consistent with ASD-related genes, and it is strongly co-expressed $\left(P<2.2 \times 10^{-16}\right.$, Wilcoxon test) with a module of genes significantly associated with ASD.
\end{abstract}

Conclusions: Rare deleterious mutations in EFR3A were found to be associated with ASD using an experiment-wide significance threshold. Synaptic phosphoinositide metabolism has been strongly implicated in syndromic forms of ASD. These data for EFR3A strengthen the evidence for the involvement of this pathway in idiopathic autism.

Keywords: Autism spectrum disorder, Genetics, Rare variants, EFR3A, Synapse, Phosphoinositide metabolism

\section{Background}

Autism spectrum disorders (ASDs) are defined by persistent deficits in social communication and social interaction and restricted repetitive patterns of behavior, interests or activities [1]. These syndromes are common in the population, with a prevalence of approximately $1 \%$ [2], and demonstrate both considerable phenotypic

\footnotetext{
* Correspondence: abha.gupta@yale.edu; matthew.state@ucsf.edu

${ }^{1}$ Department of Pediatrics and Child Study Center, Yale School of Medicine,

New Haven, CT 06520, USA

${ }^{15}$ Department of Psychiatry, University of California San Francisco, San

Francisco, CA 94143, USA

Full list of author information is available at the end of the article
}

and extensive genetic heterogeneity [3]. High-throughput sequencing approaches have provided substantial insight into the genomic architecture of ASDs. For example, multiple analyses of whole-exome sequencing data demonstrate an over-representation of de novo, loss-of-function mutations in brain-expressed genes in affected individuals and point to half a dozen new ASD genes [3-6]. These have been identified based on the clustering of mutations in the same gene in unrelated individuals, providing strong evidence for association [3]. However, a large number of compelling, rare, de novo missense mutations are also found in probands, 
though a clear threshold for identifying the association of these mutations with ASD is less obvious. Both the rarity of the individual mutations and the small size of current exome discovery cohorts suggest that clarifying which of these de novo mutations point to bona fide ASD genes will require alternative approaches. Largescale, targeted, case/control sequencing as a complement to de novo mutation discovery in ASD is one such strategy.

In a whole-exome analysis of 238 families [3], we identified a single proband carrying two novel de novo missense mutations in synaptic genes, one each in EFR3A (Eighty-five Requiring 3A [NCBI Reference Sequence: NM_015137]) and CASK (Calcium/Calmodulin-dependent Serine/Threonine Kinase [NCBI Reference Sequence: NM_003688]). Both yeast EFR3 [7] and mammalian EFR3A and EFR3B [8] have been linked to the control of phosphoinositide metabolism, a pathway demonstrated to play a role in ASD [9]. CASK is implicated in X-linked intellectual disability [10]. Neither the occurrence of one or several de novo missense mutations in a single affected individual is a statistically significant finding. However, our overall analysis of 599 simplex ASD quartets suggests that approximately $20 \%$ of de novo missense mutations in brainexpressed genes found in cases will prove to be true ASD loci, representing an approximately fourfold increase over a brain-expressed gene chosen at random [11]. Given an increased prior probability based on the exome results and the strong biological plausibility of both genes, we conducted a targeted analysis of EFR $3 A$ and CASK in large cohorts using both Sanger sequencing and whole-exome data. We found that rare deleterious mutations in EFR3A are associated with ASD using an experiment-wide significance threshold.

\section{Methods \\ Subjects}

Initial cases were drawn from the Simons Simplex Collection (SSC). The SSC is an exhaustively characterized ASD family cohort, with the majority of families consisting of a proband, two unaffected parents and an unaffected sibling. The diagnostic methodology used is well described elsewhere [12]. EFR3A and CASK were sequenced in 705 cases of European ancestry (Family Distribution List v13) based on genome-wide genotyping data (see below). Based on a preliminary analysis of the sequence data, mutation screening then focused on EFR3A, which was evaluated in several cohorts for which we had access to DNA or whole-exome sequencing results. Additional cases were drawn from the SSC $(n=452)$ and via collaboration with the ARRA Autism Sequencing Collaboration (AASC, $n=1,039$ ). All cases were identified as having European ancestry via genome-wide genotyping data. Sample characteristics and diagnostic methodology for the AASC have been described previously [5,13]. For controls, 912 were drawn from the National Institute of Neurological Disorders and Stroke (NINDS) Neurologically Normal Caucasian Control Panel (NDPT020, 079, 082, 084, 090, 093, 094, 095, 096, and 098). This set of adult subjects has a negative personal and family history (first-degree relatives) of neuropsychiatric illness. Additional controls were drawn from the AASC $(n=863)$ and from ongoing studies of non-neuropsychiatric conditions at our home institution (northern European (NE) controls, $n=1,614$ ). Again, all controls were of confirmed European ancestry. The NE and AASC controls were considered population controls since subjects with potential neuropsychiatric disorders were not excluded. This study only accessed de-identified biospecimens or sequencing data and no protected health information; it received an exemption from human subject research from the Yale Human Research Protection Program.

\section{Genotyping and ancestry matching}

1,304 SSC cases were genotyped using Human1M-Duo v1, Human1M-Duo v3 or HumanOmni2.5 BeadChips (Illumina, San Diego, CA, USA). 923 NINDS controls were genotyped using Illumina HumanOmniExpress12v1. 1,779 NE controls were genotyped using Illumina $550 \mathrm{~K}$ Single or 610 Quad v1 BeadChips. Subjects were removed because of: (1) genotyping call rate $<95 \%$, (2) discrepancy of genotyping data with recorded sex, and (3) Mendelian inconsistencies or cryptic relatedness (up to and including second-degree relatives).

For ancestry matching, Golden Helix SNP and Variation Suite v7.5.4 (Bozeman, MT, USA) was used in principal component analysis (PCA) of SSC cases, NINDS controls and NE controls using 8,210 SNPs common to all arrays and not in high linkage disequilibrium. Based on visualization of a scree plot (Additional file 1: Figure S1), eigenvalues of the first three principal components, which contributed the greatest amount of variation relative to the other principal components, were plotted against one another (Additional file 2: Figure S2). The interquartile range (IQR) distance around the median of the study population cluster was calculated. A threshold that included all NINDS and NE controls was determined to lie at 5 IQRs from the third quartile, and 54 SSC cases beyond this threshold were excluded as ancestral outliers (Additional file 3: Figure S3). The final cohort sizes were 1,157 SSC cases, 912 NINDS controls and 1,614 NE controls.

AASC cases and controls were genotyped using Illumina microarrays, including $550 \mathrm{~K}, 610 \mathrm{~K}$ and $1 \mathrm{M}$ BeadChips, and also filtered to exclude subjects because of: (1) genotyping call rate $<95 \%$, (2) discrepancy of genotyping data with recorded sex, and (3) Mendelian inconsistencies or cryptic relatedness. Ancestry matching between AASC 
cases and controls was conducted using PCA of genotyping data for a subset of SNPs common to all arrays; each case was matched to the nearest control using a greedy algorithm. The final cohort sizes were 1,039 cases and 863 controls, all of European descent.

\section{Sanger sequencing of Simons Simplex Collection cases and NINDS controls}

PCR primers were designed to flank all coding exons and splice sites of EFR3A and CASK (Additional file 4: Table S1). Then $10 \mathrm{ng}$ lymphoblastoid cell line-derived genomic DNA served as template in a $25 \mu \mathrm{l}$ PCR containing $1 \times$ PreMix D buffer (Epicentre Biotechnologies, Madison, WI, USA), $0.48 \mu \mathrm{M}$ each forward and reverse primer, and $0.36 \mu \mathrm{L}$ Taq polymerase/0.072 $\mu \mathrm{L}$ Pyrococcus furiosus (PFU) polymerase. Both enzymes, which were synthesized in house, were used to permit proofreading during PCR and reduce Taq-induced mutations. A Tetrad2 Peltier Thermal Cycler (Bio-Rad, Hercules, CA, USA) was programmed as follows: $95.0^{\circ} \mathrm{C} / 5 \mathrm{~min} ; 40$ cycles of $95.0^{\circ} \mathrm{C} / 30 \mathrm{sec}, 60.0^{\circ} \mathrm{C} / 30 \mathrm{sec}$ and $72.0^{\circ} \mathrm{C} / 60 \mathrm{sec} ; 72.0^{\circ} \mathrm{C} /$ $10 \mathrm{~min}$. PCR products were visualized by agarose gel electrophoresis and sent to Beckman Coulter Genomics (Danvers, MA, USA) or the Yale Keck Biotechnology Resource Laboratory (New Haven, CT, USA) for Sanger sequencing. Chromatograms were aligned and analyzed using Sequencher v4.9 (Gene Codes, Ann Arbor, MI, USA). We obtained a $96 \%$ sequencing success rate for both cases and controls. All potential rare ( $<1 \%$ frequency) nonsynonymous variants were confirmed by a second round of PCR and Sanger sequencing in forward and reverse directions, using blood-derived genomic DNA for SSC cases since it was available. Segregation analysis of confirmed variants was performed using blood-derived genomic DNA from all family members, which were only available for SSC cases.

\section{Whole-exome data from northern European controls and ARRA Autism Sequencing Collaboration cases/controls}

For the NE controls, we examined whole-exome sequencing data. Greater than $98 \%$ of the EFR3A coding and splice site sequence was covered by at least eight independent reads. For variant calling, a minimum read threshold of only one independent read was used to minimize the liability for false negatives. All coding and splice site variants with a SAMtools SNP quality score $\geq 50$ were subjected to confirmation by PCR and Sanger sequencing of whole-genome amplified DNA.

We also examined whole-exome sequencing data for AASC cases and controls (generated by the Broad Institute and Baylor College of Medicine), which were treated as a matched set and subjected to identical quality control and variant calling criteria within each site. All coding and splice site variants were identified after three rounds of filtering the whole-exome data for quality control. Variants were excluded if: (1) they had $\geq 10 \%$ missing calls, (2) they had average coverage $<17$ for Broad cases/ controls and $<12$ for Baylor cases/controls, and (3) $>50 \%$ of minor allele calls had $<17$ reads or a balance of depth $>0.66$ for Broad cases/controls and $<12$ reads or a balance of depth $>0.75$ for Baylor cases/controls (balance of depth being defined as the number of reference reads divided by the total number of reads). Filtering criteria differed between the two sites since samples were sequenced on different platforms and the data were processed using different software packages (Illumina/GATK at Broad and Solid/AtlasSNP2 at Baylor). AASC case and control variants were not confirmed by Sanger sequencing. However, given that the cohorts are approximately the same size and the entire AASC set was subjected to identical sequencing methods, we anticipated that calling errors would be randomly distributed across affected and unaffected individuals.

To assess the novel singleton status of variants identified in all case and control groups, we queried dbSNP137 and whole-exome sequencing data from an additional 6,503 individuals from release ESP6500 of the Exome Variant Server, comprising 4,300 European-Americans and 2,203 African-Americans.

\section{Analysis by conservation measures}

We evaluated conservation at the positions of novel nonsynonymous singleton mutations in EFR3A with three widely used informatics tools: PhyloP (phylogenetic $P$ values), GERP (genomic evolutionary rate profiling) and ConSurf. PhyloP scores [14] were obtained from the UCSC Genome Browser. A PhyloP score $\geq 1.3$ indicates $P=0.05$ for conservation and was used as a threshold to determine whether a mutation occurred at a conserved site. GERP scores [15] were obtained from the SeattleSeq annotation pipeline. A GERP score $\geq 5$ was used as a threshold to determine conservation [16]. Regarding ConSurf analysis, a multiple EFR3 protein sequence alignment was constructed using PSI-Blast, which was then edited to remove partial or redundant sequences and produce a comprehensive sampling of genetic space. Both EFR3A and EFR3B were included to increase the number of sequences available, which totaled 42 (Additional file 5: Figure S4). The alignment was produced with TCoffee and sent to the ConSurf server to quantify conservation [17]. The server normalizes the conservation score for each amino acid such that average positions cluster around zero; the most conserved residues have negative scores, and the least conserved are positive.

\section{Structure-based analysis}

The crystal structure of the N-terminal fragment (amino acids 8 to 562) of the Saccharomyces cerevisiae Efr3 was 
recently determined [18]. Yeast Efr3 and human EFR3A were aligned through amino acid 451, corresponding to the most conserved portion of Efr3 (Additional file 6: Figure S5). We created a homology model and found that secondary structure predictions of the human EFR3A matched well with the observed secondary structure of the yeast protein. Based on the crystal structure, human EFR3A case and control mutations were blindly assessed for their potential to disrupt protein structure and function using the following structural criteria prioritization: first, it was determined whether the mutated residue was located in the protein core or on the surface as shown by the crystal. If the mutation was located in the core, it was then assessed, taking secondary structure into account, for whether a hydrophilic residue would be placed in a hydrophobic environment or whether the mutation changed the residue size, which could result in a defect in packing the core or misfolding. If the mutation was located on the surface of the protein, it was then determined whether that area was well conserved and hence likely to be functionally important. If so, any change in residue charge and/or size was categorized as potentially disruptive as these could affect protein-protein or protein-membrane interactions. To this end we devised a grading scheme, where deleterious variants received a score of 3 or higher. It should be noted, however, that this grading scheme cannot take into account interactions of EFR3A that have not been described to date.

\section{In situ hybridization}

Human brain tissue samples were fixed in 4\% PFA (Paraformaldehyde) at $4^{\circ} \mathrm{C}$ for 2 to 3 days, cryoprotected in graded sucrose solutions (up to $30 \%$ ) at $4^{\circ} \mathrm{C}$, frozen at $-40^{\circ} \mathrm{C}$ in isopentane/dry ice, and stored at $-80^{\circ} \mathrm{C}$. Frozen samples were cut at $20 \mu \mathrm{m}$ using a Leica CM3050S cryostat and mounted onto gelatine-coated slides. To prepare complementary RNA probes, cDNA was amplified with T7 and SP6 promoter-attached primers (T7/forward primer: TAATACGACTCACTATAGGGAGACGGGCCA CCATTTGGGAACCT, SP6/reverse primer: GCGAT TTAGGTGACACTATAGCCAGCACTGTCGGACCTAT GGA) and used to generate digoxigenin-labeled riboprobes with T7 RNA polymerase (Roche, Basel, Switzerland) for the sense probe (negative control) and SP6 RNA polymerase (Roche) for the antisense probe. After acetylation, sections were hybridized with the riboprobes at $55^{\circ} \mathrm{C} / 16 \mathrm{hr}$. They were then processed as follows: (1) rinsed in $2 \times$ SSC, (2) incubated with $20 \mu \mathrm{g} / \mathrm{ml}$ RNase A at $37^{\circ} \mathrm{C} / 30 \mathrm{~min}$, (3) washed in high stringency conditions at $60^{\circ} \mathrm{C}$, (4) incubated at room temperature (RT)/2 hr with AP-coupled anti-digoxigenin Fab fragment (Roche) in 1\% donkey serum in TBST, and (5) washed in NTMT buffer $(2 \times$ $10 \mathrm{~min})$. Signals were developed in a light-protected humidified chamber with NBT/BCIP in NTMT buffer/ $2 \mathrm{mM}$ levamisole solution at RT overnight. The sections were rinsed in TE and cover-slipped using a crystal aqueous mounting medium (Accurate Chemical and Scientific Corporation, Westbury, NY, USA). SSC: saline-sodium citrate buffer, AP: alkaline phosphatase, TBST: Tris-buffered saline, $0.1 \%$ Tween-20, NTMT: $\mathrm{NaCl}+$ Tris- $\mathrm{HCl}+\mathrm{Mag}-$ nesium chloride + Tween-20, NBT: nitro-blue tetrazolium, BCIP: 5-bromo-4-chloro-3'-indolyphosphate, TE: TrisEDTA buffer.

\section{Gene co-expression analysis}

Using data from Kang et al. [19], Spearman correlation was performed between expression levels of EFR3A and M12 genes [20] and between EFR3A and all 15,132 genes expressed in the human brain [19]. Of the 432 unique genes in M12, 356 had expression data in the array platform used by Kang et al. [19], and these were used to perform the analysis (Additional file 7: Table S2). These analyses were also performed with ten additional genes: ACTB (a housekeeping gene), CHD8, DYRK1A, EFR3B (a homologue), GRIN2B, KATNAL2, NRXN1, $S C N 2 A, S H A N K 2$ and SHANK3. The median expression correlation coefficients for the 11 genes when compared to M12 and all brain-expressed genes are shown in Additional file 8: Table S3. To show the distribution of the correlation coefficients, kernel density plots were generated using the sm.density.compare function in the sm package in $\mathrm{R}$ with the smoothing parameter $h=0.1$. The entire process was repeated for an additional three modules: M2, M3 and M16 genes [21].

\section{Statistical analysis}

All $P$ values for mutation burden, conservation measures and crystal structure analysis were calculated by the Fisher exact test. We used the right-tailed test based on the hypothesis that there would be a greater number of mutations in cases versus controls and that case mutations would be more deleterious. Since we initially investigated two genes, CASK as well as EFR3A, we performed a Bonferroni correction and multiplied the $P$ value for overall mutation burden by two. The initial de novo mutation F338S identified by whole-exome sequencing was not included in calculations of overall mutation burden between cases and controls but was included when assessing the potential deleteriousness of case versus control mutations.

To study the relative enrichment of variants at conserved positions in cases and controls, we conducted the following analysis. For each novel nonsynonymous singleton variant, we used cutoff values for three conservation measures to annotate whether each variant maps to a conserved position and is, therefore, potentially deleterious: PhyloP $\geq 1.3$ (indicates $P=0.05$ for conservation), GERP $\geq 5$ [16] and ConSurf $<0$ (indicates conservation). We also performed a 
permutation test by first creating an input file (Additional file 9: Table S4) of binary entries, with ' 1 ' indicating that the variant met the cutoff and is functional by that conservation measure and ' 0 ' indicating that it did not. For each measure, we calculated the proportion of individuals carrying functional variants in the case and control cohorts. We then calculated the ratio of the two proportions as the relative enrichment in cases. We used the largest ratio among the three measures as the test statistic for the observed data. To estimate the statistical significance, we adopted the following permutation procedure. For each of 10,000 permutations, we permuted the case and control labels of the subjects. Based on the case and control groups defined by the permuted labels, we repeated the same relative enrichment ratio calculation and estimated a $P$ value for enrichment of deleterious mutations in cases. The nonparametric Wilcoxon test was used to calculate the $P$ value for the difference in median expression correlation coefficients between EFR3A/M12 and EFR3A/all brain-expressed genes.

\section{Western blot analysis of mouse tissues}

Female C57BL/6 mice (Jackson Laboratory, Bar Harbor, ME, USA) were euthanized at approximately 21 days after birth. Organs were isolated, homogenized in lysis buffer (1\% Triton X-100, $150 \mathrm{mM} \mathrm{NaCl}, 20 \mathrm{mM}$ Tris, $0.5 \mathrm{mM}$ EDTA (Ethylenediaminetetraacetic acid), pH 7.4, supplemented with Complete EDTA-free protease inhibitor tablet (Roche)), centrifuged for $10 \mathrm{~min}$ at $16,000 \mathrm{~g}$, and the supernatant was reserved. Protein concentrations were normalized using the bicinchoninic acid (BCA) assay (Thermo Pierce, Rockford, IL, USA). The samples were analyzed by Western blot (30 $\mu \mathrm{g}$ sample/lane), probing with anti-EFR3A (Ab2, Sigma, St Louis, MO, USA) or anti-GAPDH (1D4, GeneTex, Irvine, CA, USA) antibodies. For the EFR3A Western blot, a goat HRP (horseradish peroxidase)-conjugated anti-rabbit secondary antibody (Bio-Rad, Hercules, CA, USA) was used, and the blot was developed using SuperSignal West Pico chemiluminescent reagent (Thermo Pierce). For the GAPDH Western blot, an IRDye $800 \mathrm{CW}$-conjugated anti-mouse secondary antibody (LI-COR Biosciences, Lincoln, NE, USA) was used, and the Western blot was scanned on an Odyssey imaging system (LI-COR Biosciences).

\section{Verification of EFR3A antibody specificity}

The specificity of the EFR3A antibody (Ab2, Sigma) was verified by Western blot analysis of HeLa cell lysates treated with siRNA duplexes (Integrated DNA Technologies, Coralville, IA, USA) targeted against human EFR3A or negative control siRNA, termed NC1. The siRNA sequences are shown in Additional file 10: Table S5. HeLa cells were transfected with the appropriate siRNA duplex (from a $20 \mu \mathrm{M}$ stock in $30 \mathrm{mM}$ HEPES (4-(2-Hydroxyethyl) piperazine-1-ethanesulfonic acid, N-(2-Hydroxyethyl)piperazine- $\mathrm{N}^{\prime}$-(2-ethanesulfonic acid)), $100 \mathrm{mM}$ potassium acetate) using RNAiMAX (Invitrogen, Carlsbad, CA, USA) according to the manufacturer's instructions, and after $6 \mathrm{hr}$, the media was exchanged for regular growth media. After 3 days, the cells were collected, dissolved in lysis buffer (1\% Triton X-100, $150 \mathrm{mM} \mathrm{NaCl}, 20 \mathrm{mM}$ Tris, 0.5 mM EDTA, pH 7.4, supplemented with Complete EDTA-free protease inhibitor tablet (Roche)), centrifuged for $10 \mathrm{~min}$ at $16,000 \mathrm{~g}$, and the supernatant was reserved. Protein concentrations were normalized using the BCA assay (Thermo Pierce). The samples were analyzed by Western blot (50 $\mu \mathrm{g}$ sample/lane), probing with antiEFR3A (Ab2, Sigma) or anti- $\alpha$-tubulin (B-5-1-2, Sigma) antibodies. IRDye $800 \mathrm{CW}$-conjugated anti-rabbit and anti-mouse secondary antibodies (LI-COR Biosciences) were used, and the Western blots were scanned on an Odyssey imaging system (LI-COR Biosciences).

\section{Results}

To test our hypothesis that mutations in EFR3A and/or CASK confer risk for ASD, we performed Sanger sequencing of all coding exons and splice sites of both genes in 705 comprehensively phenotyped European cases from the SSC. All rare ( $<1 \%$ frequency) nonsynonymous variants were confirmed by a second round of Sanger sequencing. We focused on novel alleles seen only once (singleton variants) and not present in two large databases, dbSNP137 and Exome Variant Server, the latter of which had 6,503 exomes. We reasoned that this strategy would most likely identify deleterious substitutions subject to purifying selection and provide, along with case/control matching for ancestry, the most robust protection against population stratification [22].

In CASK, only two variants met these criteria among all 705 cases (Additional file 11: Table S6). In light of the low cumulative allele frequency and anticipated low power to detect an effect, we did not pursue this gene further. We identified six novel nonsynonymous singleton mutations in EFR3A (Table 1 and Additional file 12: Table S7) and, consequently, we proceeded to screen this gene in several cohorts for which we had access to DNA or whole-exome sequencing data. We identified an additional 1,491 European cases: (1) 452 from the SSC were subjected to Sanger sequencing and (2) 1,039 from the AASC had whole-exome sequencing data, for a total of 2,196 cases. We identified a total of 3,389 European controls: (1) 912 NINDS neurologically normal European controls matched to SSC cases via PCA of genotyping data and subjected to Sanger sequencing, (2) 1,614 neuropsychiatrically unscreened controls of NE origin matched to SSC cases and who had whole-exome sequencing data, and (3) 863 from the AASC with whole-exome sequencing data. For the NE control exomes, a minimum read 
Table 1 Novel nonsynonymous singleton mutations in EFR3A

\begin{tabular}{|c|c|c|c|c|c|c|c|c|c|c|c|c|c|c|c|}
\hline Cohort & Exon & Amino acid change $^{a}$ & Chromosome & hg19 & Reference & Variant & ID & Sex & Father & Mother & Sib & PhyloP & GERP & ConSurf & Crystal \\
\hline \multirow[t]{20}{*}{ Cases $(n=2,196)$} & \multicolumn{15}{|c|}{ SSC cases $(n=705)$} \\
\hline & 3 & R70C & 8 & 132957112 & C & $\mathrm{T}$ & 12093.p1 & M & + & - & $\mathrm{n} / \mathrm{a}$ & 2.95617 & 5.73 & -1.51 & Deleterious \\
\hline & 4 & L118P & 8 & 132958867 & T & C & 12610.p1 & M & + & - & $-F$ & 3.21686 & 5.47 & -0.73 & Deleterious \\
\hline & 7 & G243A & 8 & 132968104 & G & C & 11572.p1 & M & - & + & $+F$ & 6.387 & 5.64 & -0.291 & Benign \\
\hline & 10 & $\mathrm{~F} 338 \mathrm{~S}^{\mathrm{b}}$ & 8 & 132982744 & T & C & 11379.p1 & $\mathrm{F}$ & - & - & $-F$ & 4.58456 & 5.54 & -1.107 & Deleterious \\
\hline & 15 & I534T & 8 & 132996411 & T & C & 11473.p1 & M & - & + & $+F$ & 4.78917 & 6.02 & -0.113 & $\mathrm{n} / \mathrm{a}$ \\
\hline & 15 & |576_A577ins| $\left.\right|^{c}$ & 8 & 132996539 & * & l:ATT & 11577.p1 & M & - & + & $+F$ & 3.628 & 5.03 & 0.043 & $\mathrm{n} / \mathrm{a}$ \\
\hline & 22 & $\mathrm{~T} 785 \mathrm{~A}$ & 8 & 133015525 & A & G & 11808.p1 & $\mathrm{F}$ & - & + & $-F$ & 3.02491 & 4.27 & -0.312 & $\mathrm{n} / \mathrm{a}$ \\
\hline & \multicolumn{15}{|c|}{ SSC cases $(n=452)$} \\
\hline & 5 & F123L & 8 & 132962216 & T & $C$ & 13507.p1 & M & - & + & $-M$ & 4.86423 & 5.51 & -1.377 & Deleterious \\
\hline & 7 & G216Sfs*12 & 8 & 132968022 & * & I:TCGCATA & 11027.p1 & M & - & + & $-M$ & 6.995 & 6.07 & -1.591 & Deleterious \\
\hline & \multicolumn{15}{|c|}{ AASC cases $(n=1039)$} \\
\hline & 3 & K50E & 8 & 132957052 & A & G & 20094 & M & & $\mathrm{n} / \mathrm{a}$ & & 5.32367 & 5.55 & -1.59 & Deleterious \\
\hline & 9 & A321S & 8 & 132980647 & G & $\mathrm{T}$ & OOHI1409C & M & & $\mathrm{n} / \mathrm{a}$ & & 3.10583 & 5.51 & -0.66 & Benign \\
\hline & 10 & V337L & 8 & 132982740 & G & C & OOHI1533A & M & & $\mathrm{n} / \mathrm{a}$ & & 6.32607 & 5.54 & -0.986 & Benign \\
\hline & 14 & D504G & 8 & 132991604 & A & G & 06C57233 & M & & $\mathrm{n} / \mathrm{a}$ & & 4.41785 & 5.06 & -0.002 & $\mathrm{n} / \mathrm{a}$ \\
\hline & 14 & L508P & 8 & 132991616 & T & C & $07 C 71126$ & M & & $\mathrm{n} / \mathrm{a}$ & & 4.19838 & 5.06 & 0.349 & n/a \\
\hline & 14 & I510V & 8 & 132991621 & A & G & 20072 & M & & $\mathrm{n} / \mathrm{a}$ & & 2.07681 & 3.88 & 0.088 & $\mathrm{n} / \mathrm{a}$ \\
\hline & 15 & Q528R & 8 & 132996393 & A & G & $98 \mathrm{HI} 0204 \mathrm{~A}$ & M & & $\mathrm{n} / \mathrm{a}$ & & 5.21937 & 6.02 & 0.08 & $n / a$ \\
\hline & 17 & M646V & 8 & 132998507 & A & G & 08C73985A & M & & $\mathrm{n} / \mathrm{a}$ & & 2.13232 & 4.65 & 2.129 & $\mathrm{n} / \mathrm{a}$ \\
\hline \multirow[t]{10}{*}{ Controls $(n=3,389)$} & \multicolumn{15}{|c|}{ NINDS controls $(n=912)$} \\
\hline & 2 & P14R & 8 & 132952776 & C & G & ND11540 & M & & $\mathrm{n} / \mathrm{a}$ & & 5.86267 & 5.93 & -1.264 & Benign \\
\hline & \multicolumn{15}{|c|}{ NE controls $(n=1,614)$} \\
\hline & 5 & $\mathrm{R} 161^{* d}$ & 8 & 132962330 & C & T & S19G8 & M & & $\mathrm{n} / \mathrm{a}$ & & 6.995 & 6.07 & -1.591 & Deleterious \\
\hline & 6 & M194V & 8 & 132966156 & A & G & $\mathrm{S} 16 \mathrm{H} 7$ & $\mathrm{~F}$ & & $\mathrm{n} / \mathrm{a}$ & & 5.34254 & 5.73 & -0.985 & Benign \\
\hline & 9 & E320D & 8 & 132980646 & G & $\mathrm{T}$ & S6G2 & $\mathrm{F}$ & & $\mathrm{n} / \mathrm{a}$ & & 1.06245 & 2.3 & -0.162 & Benign \\
\hline & 10 & N354D & 8 & 132982791 & A & G & S6B11 & M & & $\mathrm{n} / \mathrm{a}$ & & 0.272244 & 0.403 & 1.188 & Benign \\
\hline & 13 & T451M & 8 & 132991119 & C & T & $\mathrm{S} 4 \mathrm{H} 5$ & M & & $\mathrm{n} / \mathrm{a}$ & & 2.72643 & 5.71 & 0.289 & Benign \\
\hline & 15 & R532W & 8 & 132996404 & C & $\mathrm{T}$ & S1B3 & $\mathrm{F}$ & & n/a & & 0.659283 & 0.794 & 1.218 & $\mathrm{n} / \mathrm{a}$ \\
\hline & 15 & D570G & 8 & 132996519 & A & G & S15E2 & $\mathrm{F}$ & & $\mathrm{n} / \mathrm{a}$ & & 5.21937 & 6.02 & -0.169 & $n / a$ \\
\hline
\end{tabular}


Table 1 Novel nonsynonymous singleton mutations in EFR3A (Continued)

\begin{tabular}{|c|c|c|c|c|c|c|c|c|c|c|c|c|}
\hline \multicolumn{13}{|c|}{ AASC controls $(n=863)$} \\
\hline 3 & G55C & 8 & 132957067 & G & $\mathrm{T}$ & 04C27095A & $\mathrm{F}$ & $\mathrm{n} / \mathrm{a}$ & 6.81431 & 5.73 & -1.321 & Benign \\
\hline 4 & F100L & 8 & 132958812 & $\mathrm{~T}$ & C & 05C42103 & M & $\mathrm{n} / \mathrm{a}$ & 4.82034 & 5.47 & -0.879 & Benign \\
\hline 8 & D268G & 8 & 132971858 & A & G & $05 C 42750$ & $\mathrm{~F}$ & $\mathrm{n} / \mathrm{a}$ & 4.99605 & 5.64 & 0.396 & Benign \\
\hline 15 16 & ? (5' splice site $)^{d}$ & 8 & 132996549 & $\mathrm{~T}$ & C & 05C45515 & $\mathrm{F}$ & $n / a$ & 6.995 & 6.07 & -1.591 & $\mathrm{n} / \mathrm{a}$ \\
\hline
\end{tabular}

${ }^{\mathrm{a} A l l}$ mutations are single alleles, i.e., heterozygous.
${ }^{\mathrm{b}} \mathrm{F} 338 \mathrm{~S}$ is the initial de novo mutation identified by whole-exome sequencing.

For 1576_A577insl, PhyloP, GERP and ConSurf scores were derived by averaging the scores of the adjacent positions.

${ }^{\mathrm{d}}$ These mutations were assigned the maximum conservation scores found for any position in EFR3A.

*Under the Reference column indicates absence of the insertion/deletion variant.

AASC, ARRA Autism Sequencing Collaboration; F, female; GERP, genomic evolutionary rate profiling; M, male; n/a, not applicable; NE, northern European; NINDS, National Institute of Neurological Disorders and Stroke; PhyloP, phylogenetic $P$ values; SSC, Simons Simplex Collection. 
threshold of only one independent read was used to identify variants in an effort to minimize false negatives. For the AASC dataset, Broad cases/controls and Baylor cases/controls were matched and evaluated using identical variant-calling approaches and filtering criteria within each site. All rare nonsynonymous variants in SSC cases, NINDS controls and NE controls were confirmed by Sanger sequencing; confirmations were not available for case and control AASC variants.

The analysis of a total of 2,196 cases and 3,389 controls demonstrated that novel nonsynonymous singleton mutations in EFR3A were twice as frequent in cases compared to controls. However, the $P$ value was not statistically significant when corrected for the investigation of two genes since we initially analyzed CASK as well as EFR3A $(16 / 2,196$ cases and $12 / 3,389$ controls; $P=0.084$, odds ratio $=2.065,95 \%$ confidence interval $=0.924$ to 4.652 , Fisher exact test, right-tailed). Since the combination of low allele frequency and high conservation has been shown to provide high sensitivity and specificity for predicting functionality in rare variant studies, in contrast to in silico prediction programs [23], we evaluated conservation with three widely used informatics tools: PhyloP, GERP and ConSurf. All found significantly more case variants mapping to conserved positions (Tables 1 and 2). Furthermore, using 10,000 permutations of the cases and controls to test the significance of the enrichment of deleterious mutations in cases, we calculated a $P$ value of 0.0077. We evaluated family data from SSC subjects and found that all newly identified variants were transmitted. Whole-exome data for only two of these subjects (11379. p1 and 11808.p1) have now been reported; neither has a de novo loss-of-function mutation which might contribute to their phenotype. SSC case 11808.p1 does have a novel de novo missense mutation (N160S) in DGCR14 (DiGeorge Syndrome Critical Region Gene 14), which has not been associated with ASD or intellectual disability [24]. We also determined that all of the SSC subjects except one (13507. p1) have undergone genome-wide copy number variant
(CNV) analysis; none have de novo CNVs that might better explain their phenotype [25].

We took advantage of the recently available crystal structure (Protein Data Bank ID 4N5A) of the N-terminal fragment of S. cerevisiae Efr3 [18] to map the human mutations (Figure 1) and determine their potential to disrupt protein structure and function, blinded to case/control status. Because the C-terminal portion is not well conserved, only mutations up to and including amino acid 451 could be evaluated with high confidence. (The full length EFR3A protein has 821 amino acid residues.) Every mutation that was assessed to be deleterious as informed by the crystal structure was a case variant, except R161*, which is assumed to be damaging (Table 1 and Additional file 13: Table S8). R161* was found in an NE control for whom neuropsychiatric information is unavailable, so we cannot determine if this mutation is associated with any neuropsychiatric condition. Thus, crystal structure analysis also identifies a significantly greater number of deleterious mutations in cases than controls $(P=0.017$, odds ratio $=9.282,95 \%$ confidence interval $=1.119$ to 204.784 , Table 2). As would be expected, all of these deleterious mutations are also at highly conserved positions as per PhyloP, GERP and ConSurf. Interestingly, the reverse is not always true, i.e., there are mutations at highly conserved positions which were assessed to be benign in light of the crystal structure. Therefore, having knowledge of the three-dimensional structure of Efr3 enriches our analysis by providing more biological information to evaluate the deleteriousness of mutations. We also noted for subjects with family data, deleterious mutations as per the crystal structure are not shared by the unaffected siblings (Table 1).

$E F R 3 A$ is a member of the EFR3 family of genes, conserved throughout eukaryotes and essential for viability [7]. The Drosophila melanogaster homologue, rolling blackout (RBO), is highly expressed in the nervous system [26], is enriched at the neural synapse [27], and was proposed to regulate phospholipase $\mathrm{C}$ signaling [26]. RBO has

Table 2 Statistics for novel nonsynonymous singleton mutations in EFR3A

\begin{tabular}{|c|c|c|c|c|c|}
\hline Cohorts (n) & & PhyloP $\geq 1.3^{b}$ & GERP $\geq 5^{c}$ & ConSurf $<0^{d}$ & Deleterious by crystal \\
\hline Cases $(2,196)$ & Number of mutations ${ }^{a}$ & $17(0.77 \%)$ & $14(0.64 \%)$ & $12(0.55 \%)$ & $6(0.27 \%)$ \\
\hline \multirow[t]{4}{*}{ Controls $(3,389)$} & Number of mutations & $9(0.27 \%)$ & $9(0.27 \%)$ & $8(0.24 \%)$ & $1(0.03 \%)$ \\
\hline & $p^{e}$ & 0.006 & 0.030 & 0.049 & 0.017 \\
\hline & Odds ratio & 2.930 & 2.410 & 2.322 & 9.282 \\
\hline & 95\% confidence interval & $1.234-7.109$ & $0.979-6.032$ & $0.885-6.214$ & $1.119-204.784$ \\
\hline
\end{tabular}

${ }^{a}$ The initial de novo mutation was not included in calculations of mutation burden but was in calculations involving severity.

${ }^{b}$ PhyloP $\geq 1.3$ indicates $P=0.05$ for conservation.

${ }^{\mathrm{C}} \mathrm{GERP} \geq 5[16]$.

${ }^{\mathrm{d} C o n S u r f}<0$ indicates conservation.

${ }^{\text {e}}$ All $P$ values are calculated by Fisher exact test, right-tailed. $P \leq 0.05$ are in bold.

GERP, genomic evolutionary rate profiling; PhyloP, phylogenetic $P$ values. 


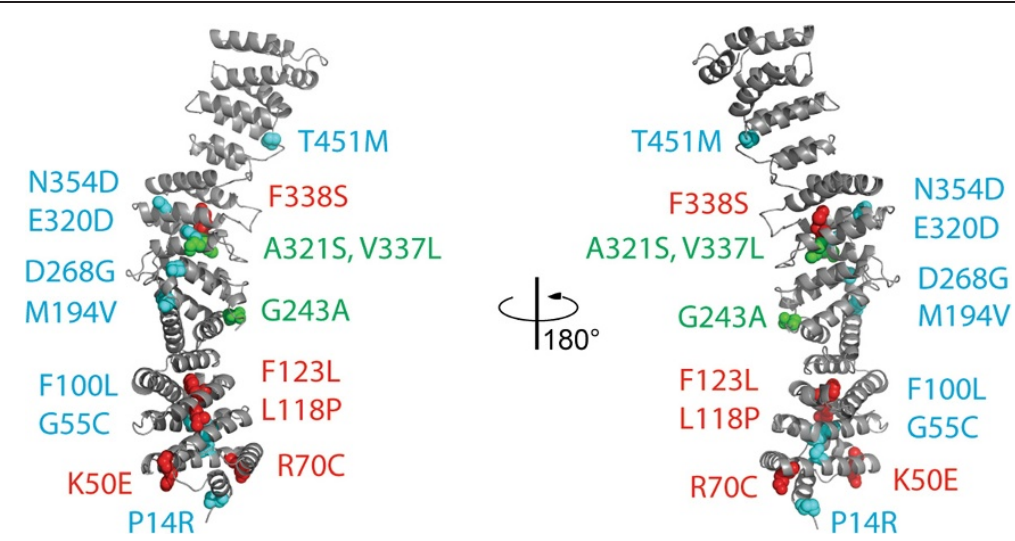

Figure 1 Ribbon diagram of S. cerevisiae Efr3 crystal structure. Crystallization of Efr3 revealed a series of HEAT repeats, as we had predicted bioinformatically. Alignment of yeast Efr3 and human EFR3A was reliable to amino acid 451 . Blinded to case/control status, the human mutations were mapped and analyzed for their potential to disrupt protein structure and function given the three-dimensional crystal structure. Mutations in red are deleterious and found in cases. (R161* in a control and G216Sfs*12 in a case are not shown but presumed to be deleterious.) Mutations in green are benign and found in cases. Mutations in blue are benign and found in controls.

also been proposed to function as a transmembrane lipase [26], but structural analysis of Efr3 does not support this hypothesis (Additional file 14: Table S9) [18]. Instead, it shows that EFR3/RBO has a scaffold function with the majority of the protein comprising alpha-helical HEAT (Huntington, Elongation factor 3, regulatory subunit A of protein phosphatase $2 \mathrm{~A}$, and Target of rapamycin) repeats.

The tissue expression of EFR3A has not been described, so we performed Western blot analysis of several mouse tissues and found that EFR3A is broadly expressed, including in the brain (Additional file 15: Figure S6). We also analyzed its expression using exon-array data from a study of the spatio-temporal transcriptome of the human brain [19]. There is a steady increase in EFR3A mRNA levels in multiple brain regions through fetal development and into adolescence (Figure 2A). In situ hybridization of adult human dorsolateral prefrontal cortex revealed the presence of EFR $3 A$ in cortical neurons including pyramidal neurons (Figure 2B). This pattern is consistent with prior data on the expression of ASD genes [9,19], as well as functional annotation of genes that are highly co-expressed with ASD genes, showing enrichment for a category associated with the development of cortical projection (pyramidal) neurons [19].

We next identified the top 100 genes co-expressed with EFR3A (Additional file 16: Table S10) using the same dataset [19]. Gene ontology enrichment analysis using the Database for Annotation, Visualization and Integrated Discovery (DAVID v6.7) $[28,29]$ revealed synaptic genes, including SYNJ1, the major $\operatorname{PtdIns}(4,5) \mathrm{P}_{2}$ phosphatase in the brain [30], as the most significant finding (Figure 2C). We also compared the expression profile of EFR3A with a discrete module of co-expressed genes (M12) significantly associated with ASD in a prior transcriptome analysis of post-mortem autism and control brains [20]. M12 is enriched for genes involved in synaptic function, vesicular transport and neuronal projection and is downregulated in the autistic brain. We compared the distribution of expression correlation coefficients between EFR $3 A$ and M12 genes (Figure 2D) and between EFR3A and all brainexpressed genes [19] (Figure 2E). We found that the distribution between EFR3A and M12 genes was significantly skewed toward positive correlation coefficients compared to the distribution between $E F R 3 A$ and all brain-expressed genes $\left(P<2.2 \times 10^{-16}\right.$, Wilcoxon test). When a similar analysis was performed on the homologue EFR3B (which is largely brain-expressed) and eight genes strongly associated with ASD from recent CNV and exome studies [3-6,25], EFR3A was the most strongly correlated with M12 expression (Figure 2D). We repeated this process with three additional modules of co-expressed genes (M2, M3 and M16) identified by a prior analysis of BrainSpan transcriptome data from normal brains [21]. All are significantly associated with ASD candidate genes, although M2 and M3 are enriched for early fetal transcriptional regulators affected by de novo loss-of-function mutations in ASD, while M16, which has significant overlap with M12, is enriched for synaptic genes upregulated during late fetal/early postnatal stages and genes harboring inherited common variants in ASD. As might be expected given its developmental expression pattern and synaptic function, EFR3A is positively correlated with M16 (Additional file 17: Figure S7A; $P<2.2 \times 10^{-16}$, Wilcoxon test) and negatively correlated with M2 and M3 (Additional file 17: Figure S7B,C; $P<2.2 \times 10^{-16}$, Wilcoxon test).

\section{Discussion}

Our conservation, structure-based functional and expression analyses suggest a role for rare deleterious EFR $3 A$ 

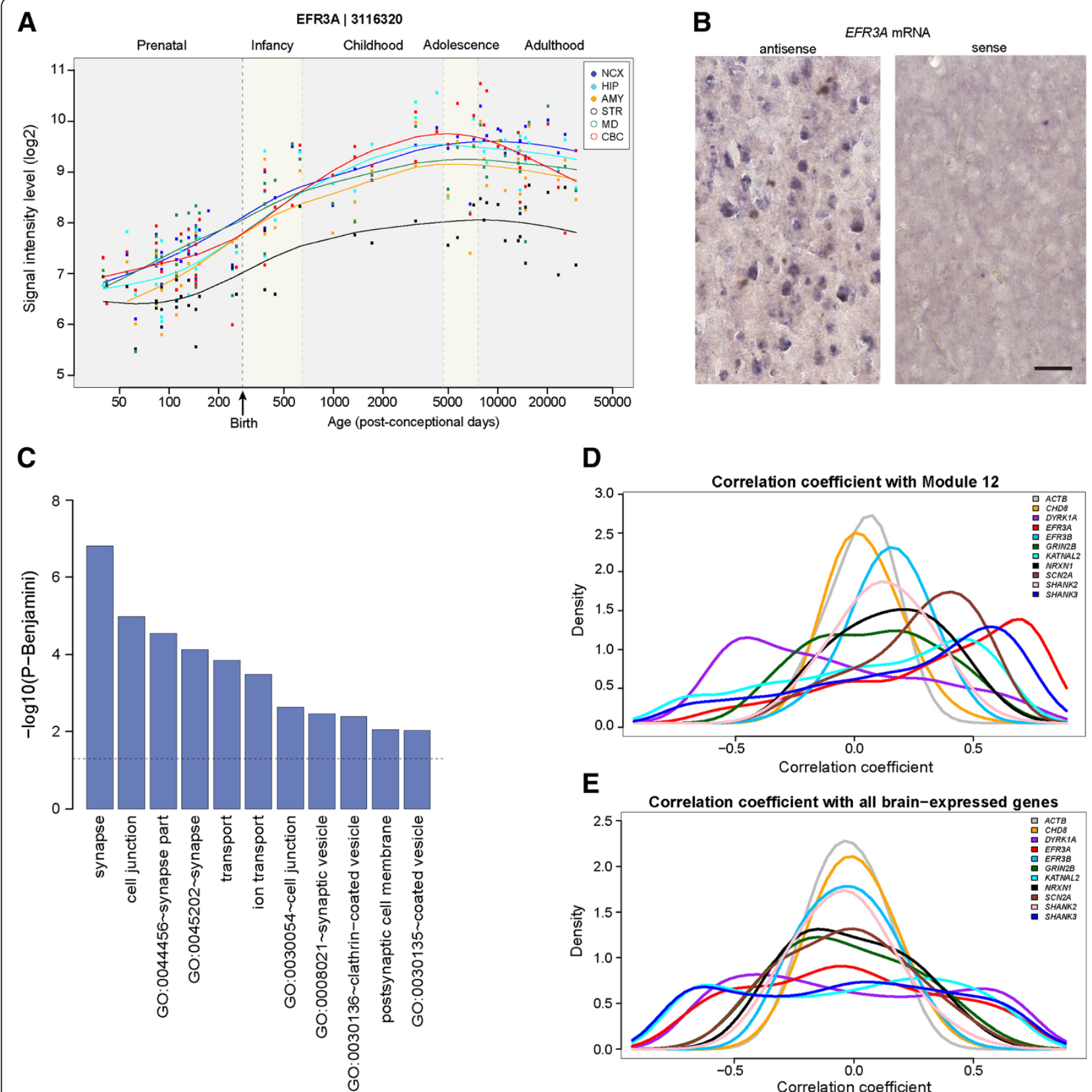

E

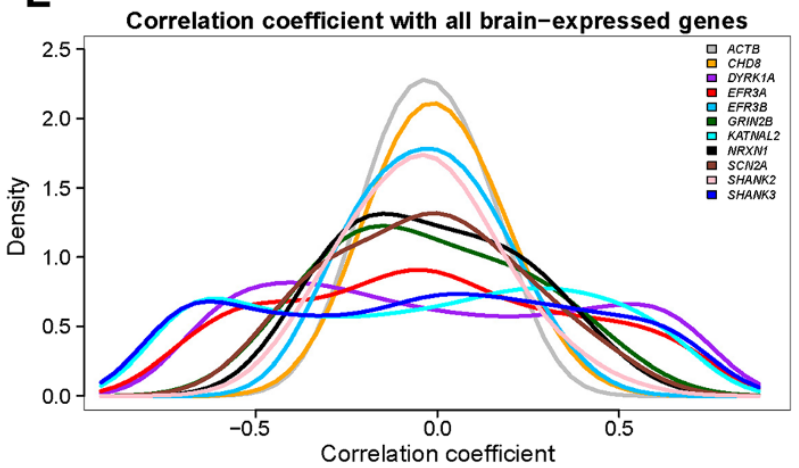

Figure 2 Expression analysis of human EFR3A. (A) Spatio-temporal mRNA expression of EFR3A in the human brain. Line plots show log 2 -transformed exon-array signal intensity during prenatal to adult stages. (B) In situ hybridization of EFR3A, using antisense and sense (negative control) probes, in the dorsolateral prefrontal cortex of 40-year-old human brain. Scale bar, 20 Mm. (C) Functional annotation of top 100 genes correlated with EFR3A expression. The dashed line is the threshold for significance, $P=0.05$. (D) Distribution of expression correlation coefficients of EFR3A and ASD genes with M12 genes $(n=356)$ [20]. (E) Distribution of expression correlation coefficients of EFR3A and ASD genes with all brain-expressed genes $(n=15,132)[19]$. The homologue EFR3B is shown for comparison and ACTB, a housekeeping gene, is included as a negative control.

mutations in the risk for ASD, adding to the emerging data on specific synaptic functions, including phosphoinositide metabolism, relevant to these disorders. Multiple resequencing projects for ASD have revealed numerous rare variants in both cases and controls. Given the overrepresentation of de novo loss-of-function mutations in cases, it is implausible that a subset of damaging missense mutations does not carry risk as well. However, differentiating relevant functional mutations from the large collection of neutral background variation remains a challenge. We have approached this issue by following up an observation of a de novo mutation in an ASD proband with a relatively large case/control analysis, relying on diverse approaches to identify putatively deleterious variants. While 
the overall burden of singleton variants was not impressive, the use of multiple conservation measures and crystal structure analysis to segregate functional variation showed consistent evidence for experiment-wide association with ASD.

Our results would clearly not survive correction for genome-wide comparisons. Of course, given the distribution of singleton mutations across the genome, this statistical threshold, if applied to every targeted analysis, would demand implausibly large case/control samples. In an effort to skirt this problem, we used an initial observation in an unbiased exome-wide study to establish a narrow hypothesis and then relied on an experimentwide $P$ value threshold for our case/control analysis. At present, this seems a reasonable approach to evaluating single gene association. Additional data on the distribution of de novo missense mutations in the genome and the integration of ASD risk associated with varying classes of mutations [31] with co-expression network data $[11,21]$ will shed significant light on the contribution of any one gene to ASD.

Our expression data, combined with evidence for the involvement of EFR3A in synaptic phosphoinositide metabolism [8], suggest that EFR3A may play an important role in synaptic function during human fetal brain development. In addition to the significant conservation and structure-based findings, our analysis comparing the expression profile of EFR3A with M12 and M16 further suggests that this gene is associated with ASD. Not only are EFR3A and M12/M16 expression strongly correlated but $E F R 3 A$ is also the most strongly correlated in the context of ASD-associated genes and its homologue EFR3B. Although co-expression data do not prove that a gene causes a disorder, they can provide another piece of supportive evidence $[11,21]$. The determination of when in development and in what cell types EFR3A is expressed provides insight into how EFR3A mutations might contribute to the pathophysiology of ASD.

A potential limitation of this study is that we combined data from Sanger sequencing (SSC cases and NINDS controls) and whole-exome sequencing (AASC cases/controls and NE controls). It is possible that the two techniques can yield different sets of variants. As described under Methods, for the NE controls, we determined that $>98 \%$ of the coding and splice site sequences were covered by at least eight independent reads. To minimize false negatives in controls that might bias toward an excess of rare mutations in cases, a minimum of only one independent read was used to identify variants for confirmation. Regarding the AASC samples, case and control exome data were subjected to identical variant-calling approaches and filtering criteria within each site and were, therefore, treated equally, suggesting that any error should be randomly distributed between these groups.
Another limitation is that we did not find additional de novo mutations in the subjects for whom family DNA was available (only SSC cases), which would strengthen the association of EFR3A mutations with ASD. However, there is abundant evidence that inherited mutations also contribute to ASD [31]. The presence of SSC case mutations in unaffected parents and/or siblings points to incomplete penetrance, as expected in complex genetic disorders such as ASD. The crystal structure analysis was able to stratify the mutations further by determining that potentially deleterious variants were generally not shared by siblings. Although this is an interesting observation, it is based on a very small number of events (five SSC case mutations with both sibling data and crystal structure information) and cannot be assigned statistical significance. We did observe one premature stop codon mutation in an NE control as well as in an SSC case, indicating that EFR3A mutations are not sufficient to cause ASD. However, neuropsychiatric data was not available for this control cohort. Moreover, the identification of well-established ASD-associated variants in unscreened controls is so commonplace as to be expected in a study such as this one.

EFR3A is a critical component of a complex containing a phosphatidylinositol 4-kinase that synthesizes the plasma membrane pool of the phosphoinositide PtdIns4P, the direct precursor of $\operatorname{PtdIns}(4,5) \mathrm{P}_{2}[8]$. PtdIns $(4,5) \mathrm{P}_{2}$ has a wide variety of direct functions in the central nervous system, including regulation of exo/endocytosis, ion channel function, neurotransmitter receptors, and transporters and nucleation of the actin cytoskeleton [32,33]. Additionally, PtdIns $(4,5) \mathrm{P}_{2}$ is a precursor to numerous signaling metabolites: diacylglycerol and $\mathrm{Ins}_{3}$ (via phospholipase $\mathrm{C}$ activity), which are key regulators of $\mathrm{Ca}^{2+}$ signaling, and PtdIns $(3,4,5) \mathrm{P}_{3}$ (via PI 3-kinase activity), which mediates many cellular processes such as activation of the Akt/ mTOR signaling pathway [30]. Mutations in PTEN, which encodes a PtdIns $(3,4,5) \mathrm{P}_{3}$ phosphatase, and in TSC1 and TSC2, which are key effectors in the $\operatorname{PtdIns}(3,4,5) \mathrm{P}_{3}$ signaling pathway, have demonstrated the importance of synaptic phosphoinositide signaling in syndromic forms of autism (Figure 3) $[9,34,35]$. Common polymorphisms and rare CNVs in $M E T$, another gene involved in phosphoinositide metabolism, have implicated this pathway in idiopathic ASD as well [36,37].

The identification of rare deleterious mutations in EFR3A, a gene linked to PtdIns4P synthesis (Figure 3), further strengthens the role of phosphoinositide metabolism in ASD. The precise effects of EFR3A on the levels of various phosphoinositides still have to be determined, and an EFR3A knock-out mouse is not yet available. Delineating the molecular details and functional significance of interactions between EFR3A and its binding partners will allow the development of in vitro assays to assess further the 


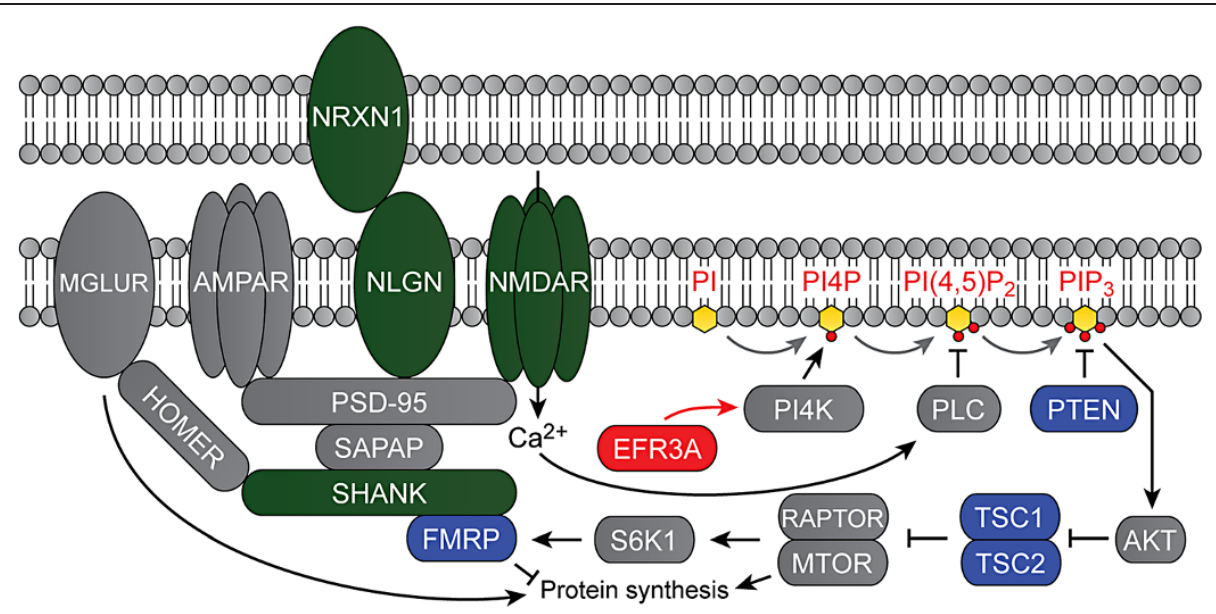

Figure 3 ASD-related molecules at the synapse. Mutations in proteins in green have been demonstrated to carry risk for idiopathic ASD, and mutations in proteins in blue cause syndromic forms of ASD. EFR3A has been linked to phosphoinositide metabolism [8].

severity of the variants we report here. Importantly, phosphoinositide metabolizing enzymes are pharmacologically targetable [38-40]. The applicability of this approach towards ASD has been shown for the closely connected mTOR pathway in mouse models of tuberous sclerosis [41,42]. Therefore, mutations in EFR3A and perturbations in phosphoinositide metabolism may point to a potential avenue for treatment in a subset of ASD patients.

\section{Conclusions}

Rare nonsynonymous mutations in EFR3A are significantly more common among ASD cases than controls at positions that are conserved and positions that would be disruptive to protein structure and function based on analysis of the Efr3 crystal structure. These results further implicate phosphoinositide metabolism in the pathophysiology of ASD, a pathway that is pharmacologically targetable. Exactly how EFR3A mutations contribute to that pathophysiology will have to await further delineation of how the protein functions and the development of specific assays to test their severity.

\section{Availability of supporting data}

NINDS Neurologically Normal Caucasian Control Panel: [http://ccr.coriell.org/Sections/Collections/NINDS/DNA Panels.aspx?PgId $=195 \&$ coll $=$ ND $/]$

AASC controls: [https://www.nimhgenetics.org/available data/controls/]

NCBI dbSNP: [http://www.ncbi.nlm.nih.gov/snp]

EVS: [http://evs.gs.washington.edu/EVS/]

UCSC Genome Browser: [http://www.genome.ucsc.edu/]

SeattleSeq: [http://snp.gs.washington.edu/SeattleSeq Annotation138/]

ConSurf: [http://consurf.tau.ac.il/]

DAVID v6.7: [http://david.abcc.ncifcrf.gov/]

\section{Additional files}

Additional file 1: Figure S1. Scree plot of the first 50 components from principal component analysis identifies three principal components that contribute the greatest amount of variation.

Additional file 2: Figure S2. Three largest principal components of genotypes for all SSC cases, NINDS controls and NE controls were plotted against one another. EV, eigenvalue; PC, principal component.

Additional file 3: Figure S3. Interquartile range (IQR) distance around the median of the study population cluster was calculated. A threshold that included all of the NINDS and NE controls was determined to lie at 5 IQRs from the third quartile, and 54 SSC cases beyond this threshold were excluded as ancestral outliers. Included samples are in blue; excluded samples (outliers) are in green. EV, eigenvalue; PC, principal component.

Additional file 4: Table S1. Primers used for PCR and Sanger sequencing.

Additional file 5: Figure S4. Multispecies protein sequence alignment of EFR3A/B for ConSurf analysis.

Additional file 6: Figure S5. Conservation structure of the EFR3A protein as determined by ConSurf.

Additional file 7: Table S2. List of the genes in M12, M16, M2 and M3 and the subset of each which is included in the exon-array platform used by Kang et al. (2011). These subsets were used for the comparisons made in Figure 2D,E, and Additional file 17: Figure S7.

Additional file 8: Table S3. Median expression correlation coefficients for ACTB, CHD8, DYRK1A, EFR3A, EFR3B, GRIN2B, KATNAL2, NRXN1, SCN2A, SHANK2 and SHANK3 compared to M12, M16, M2, M3 and all brain-expressed genes.

Additional file 9: Table S4. Binary entries used for permutation test. Additional file 10: Table S5. All rare nonsynonymous CASK variants in SSC cases.

Additional file 11: Table S6. All rare nonsynonymous EFR3A variants. Additional file 12: Table S7. Severity of novel nonsynonymous singleton EFR3A mutations informed by Efr3 crystal structure.

Additional file 13: Table S8. Molecular modeling of EFR3A protein. Molecular modeling was accomplished by inputting reference sequences into the I-TASSER [1,2], Phyre2 [3], Raptor [4] and HHpred [5] web servers. The Protein Data Bank identification codes for template structures are indicated, with the best matches for each run in bold and the error assessment for each server shown. An independent technique for 
detecting and scoring HEAT repeats was also used [6]. Using this technique, three HEAT repeats were detected with an $E$ value less than 50, the benchmark for significance. Additionally, six HEAT repeats were detected by the REP server [7].

Additional file 14: Table S9. Top 100 genes co-expressed with EFR3A. Additional file 15: Figure S6. Expression analysis of mouse EFR3A. (A) EFR3A is expressed in several mouse tissues, including the brain, as analyzed by Western blot. (B) EFR3A antibody specificity is verified by Western blot analysis of lysates from HeLa cells treated with control siRNA (-) or three different siRNA duplexes against human EFR3A. Although this antibody works well for Western blots, it does not work well for immunofluorescence, so we were not able to provide data for protein subcellular localization.

Additional file 16: Table S10. siRNA sequences used to verify EFR3A antibody specificity.

Additional file 17: Figure S7. Co-expression analysis of human EFR3A. Distribution of expression correlation coefficients of EFR3A and ASD genes with (A) M16, (B) M2 and (C) M3 genes. The homologue EFR3B is shown for comparison and ACTB, a housekeeping gene, is included as a negative control.

\section{Abbreviations}

AASC: ARRA Autism Sequencing Collaboration; ASD: autism spectrum disorder; BCA: bicinchoninic acid; CASK: Calcium/Calmodulin-dependent Serine/ Threonine Kinase; CNV: copy number variant; DAVID: Database for Annotation, Visualization and Integrated Discovery; EFR3A: Eighty-five Requiring $3 A$; GERP: genomic evolutionary rate profiling; HEAT: Huntington, Elongation factor 3 , regulatory subunit $A$ of protein phosphatase $2 A$, and Target of rapamycin; IQR: interquartile range; NE: northern European; NINDS: National Institute of Neurological Disorders and Stroke; PCA: principal component analysis; PCR: polymerase chain reaction; PhyloP: phylogenetic $P$ values; RBO: rolling blackout; RT: room temperature; siRNA: small interfering RNA; SNP: single nucleotide polymorphism; SSC: Simons Simplex Collection.

\section{Competing interests}

The authors declare that they have no competing interests.

\section{Authors' contributions}

ARG conceived and designed the study, collected and analyzed data, and wrote and gave final approval for the manuscript. HJK, MC, LL, AGE-S and BNM collected and analyzed data, and edited and gave final approval for the manuscript. MP, FC, JMB and DF collected and analyzed data, made a critical revision and gave final approval for the manuscript. TVF, JDM, LK, MJD, RPL and $\mathrm{HZ}$ analyzed the data, and edited and gave final approval for the manuscript. PDC and NS analyzed data, made a critical revision and gave final approval for the manuscript. MWS conceived and designed the study, provided financial support, analyzed data, and wrote and gave final approval for the manuscript. All authors read and approved the final manuscript.

\section{Acknowledgements}

We are very grateful to all of the families participating in the cohorts described in this paper. We thank the members of the AASC for wholeexome sequencing data and Weizhen Ji for providing the NE control samples. We greatly appreciate the expertise of Karin Reinisch and Xudong Wu in analyzing the EFR3A human mutations using their Efr3 crystal structure. We thank Bernie Devlin, Kenneth Kidd, and Ellen J Hoffman for helpful discussions and Gordon T Ober, Michael F Walker, Nicholas M DiLullo and Cynthia A Zerillo for technical assistance. This work was supported by the National Institutes of Health (grants K08MH087639 to ARG, K99HL11134001 to MC, R01MH089208 to MJD, R37NS036251 to PDC, R01GM59507 to HZ, U01MH081896 to NS, R01MH081754-04 and RC2MH089956 to MWS), the Jane Coffin Childs Fund (JMB), the Howard Hughes Medical Institute (RPL), the James S McDonnell Foundation Scholar Award (NS) and the Simons Foundation (PDC and MWS).

\section{Author details}

${ }^{1}$ Department of Pediatrics and Child Study Center, Yale School of Medicine, New Haven, CT 06520, USA. ${ }^{2}$ Scholar Rock, LLC, 300 Third Street, 4th floor, Cambridge, MA 02142, USA. ${ }^{3}$ Department of Neurobiology, Kavli Institute for
Neuroscience, Yale School of Medicine, New Haven, CT 06520, USA. ${ }^{4}$ College of Pharmacy, University of South Florida, Tampa, FL 33612, USA. ${ }^{5}$ Department of Life Science, Chung-Ang University, Seoul, Korea. ${ }^{6}$ Department of Psychiatry and Child Study Center, Yale School of Medicine, New Haven, CT 06520, USA. 7 Department of Cell Biology, Howard Hughes Medical Institute, Program in Cellular Neuroscience Neurodegeneration and Repair, Yale School of Medicine, New Haven, CT 06520, USA. ${ }^{8}$ Department of Genetics, Howard Hughes Medical Institute, Yale School of Medicine, New Haven, CT 06520, USA. ${ }^{9}$ Department of Statistics, Carnegie Mellon University, Pittsburgh, PA 15213, USA. ${ }^{10}$ Program on Neurogenetics, Child Study Center, Yale School of Medicine, New Haven, CT 06520, USA. ${ }^{11}$ Program on Neurogenetics, Child Study Center, Department of Psychiatry, Department of Genetics, Yale School of Medicine, New Haven, CT 06520, USA. ${ }^{12}$ Department of Psychiatry, University of Pittsburgh School of Medicine, Pittsburgh, PA 15213, USA. ${ }^{13}$ Program in Medical and Population Genetics, Broad Institute of Harvard and MIT, Cambridge, MA 02142, USA. ${ }^{14}$ Departments of Biostatistics and Genetics, Yale School of Medicine, New Haven, CT 06520, USA. ${ }^{15}$ Department of Psychiatry, University of California San Francisco, San Francisco, CA 94143, USA.

Received: 22 November 2013 Accepted: 28 March 2014

Published: 29 April 2014

\section{References}

1. American Psychiatric Association: Autism Spectrum Disorder. In Diagnostic and Statistical Manual of Mental Disorders. 5th edition. Washington, DC: American Psychiatric Association; 2013:50-59.

2. Autism and Developmental Disabilities Monitoring Network Surveillance Year 2008 Principal Investigators and Centers for Disease Control and Prevention: Prevalence of autism spectrum disorders - autism and developmental disabilities monitoring network, 14 sites, United States. MMWR Surveill Summ 2008, 2012(61):1-19.

3. Sanders SJ, Murtha MT, Gupta AR, Murdoch JD, Raubeson MJ, Willsey AJ, Ercan-Sencicek AG, DiLullo NM, Parikshak NN, Stein JL, Walker MF, Ober GT, Teran NA, Song Y, El-Fishawy P, Murtha R, Choi M, Overton JD, Bjornson RD, Carrierio NJ, Meyer KA, Bilguvar K, Mane SM, Sestan N, Lifton RP, Gunel M, Roeder K, Geschwind DH, Devlin B, State MW: De novo mutations revealed by whole-exome sequencing are strongly associated with autism. Nature 2012, 485:237-241.

4. Iossifov I, Ronemus M, Levy D, Wang Z, Hakker I, Rosenbaum J, Yamrom B, Lee YH, Narzisi G, Leotta A, Kendall J, Grabowska E, Ma B, Marks S, Rodgers L, Stepansky A, Troge J, Andrews P, Bekritsky M, Pradhan K, Ghiban E, Kramer M, Parla J, Demeter R, Fulton LL, Fulton RS, Magrini VJ, Ye K, Darnell JC, Darnell RB, et al: De novo gene disruptions in children on the autistic spectrum. Neuron 2012, 74:285-299.

5. Neale BM, Kou Y, Liu L, Ma'ayan A, Samocha KE, Sabo A, Lin CF, Stevens C, Wang LS, Makarov V, Polak P, Yoon S, Maguire J, Crawford EL, Campbell NG, Geller ET, Valladares O, Schafer C, Liu H, Zhao T, Cai G, Lihm J, Dannenfelser O, Jabado Z, Peralta U, Nagaswamy U, Muzny D, Reid JG, Newsham I, Wu Y, et al: Patterns and rates of exonic de novo mutations in autism spectrum disorders. Nature 2012, 485:242-245.

6. O'Roak BJ, Vives L, Girirajan S, Karakoc E, Krumm N, Coe BP, Levy R, Ko A, Lee C, Smith JD, Turner EH, Stanaway IB, Vernot B, Malig M, Baker C, Reilly B, Akey JM, Borenstein E, Rieder MJ, Nickerson DA, Bernier R, Shendure J, Eichler EE: Sporadic autism exomes reveal a highly interconnected protein network of de novo mutations. Nature 2012, 485:246-250.

7. Baird D, Stefan C, Audhya A, Weys S, Emr SD: Assembly of the Ptdlns 4-kinase Stt4 complex at the plasma membrane requires Ypp1 and Efr3. J Cell Biol 2008, 183:1061-1074.

8. Nakatsu F, Baskin JM, Chung J, Tanner LB, Shui G, Lee SY, Pirruccello M, Hao M, Ingolia NT, Wenk MR, De Camilli P: Ptdlns4P synthesis by PI4KIIla at the plasma membrane and its impact on plasma membrane identity. J Cell Biol 2012, 199:1003-1016.

9. State MW: The genetics of child psychiatric disorders: focus on autism and Tourette syndrome. Neuron 2010, 68:254-269.

10. Hackett A, Tarpey PS, Licata A, Cox J, Whibley A, Boyle J, Rogers C, Grigg J, Partington M, Stevenson RE, Tolmie J, Yates JR, Turner G, Wilson M, Futreal AP, Corbett M, Shaw M, Gecz J, Raymond FL, Stratton MR, Schwartz CE, Abidi FE: CASK mutations are frequent in males and cause $\mathrm{X}$-linked nystagmus and variable XLMR phenotypes. Eur J Hum Genet 2010, 18:544-552. 
11. Willsey AJ, Sanders SJ, Li M, Dong S, Tebbenkamp AT, Muhle RA, Reilly SK, Lin L, Fertuzinhos S, Miller JA, Murtha MT, Bichsel C, Niu W, Cotney J, Ercan-Sencicek AG, Gockley J, Gupta AR, Han W, He X, Hoffman EJ, Klei L, Lei J, Liu W, Liu L, Lu C, Xu X, Zhu Y, Mane SM, Lein ES, Wei L, et al: Co-expression networks implicate human mid-fetal deep cortical projection neurons in the pathogenesis of autism. Cell 2013, 155:997-1007.

12. Fischbach GD, Lord C: The Simons simplex collection: a resource for identification of autism genetic risk factors. Neuron 2010, 68:192-195.

13. Liu L, Sabo A, Neale BM, Nagaswamy U, Stevens C, Lim E, Bodea CA, Muzny D, Reid JG, Banks E, Coon H, Depristo M, Dinh H, Fennel T, Flannick J, Gabriel S, Garimella K, Gross S, Hawes A, Lewis L, Makarov V, Maguire J, Newsham I, Poplin R, Ripke S, Shair K, Samocha KE, Wu Y, Boerwinkle E, Buxbaum JD, et al: Analysis of rare, exonic variation amongst subjects with autism spectrum disorders and population controls. PLOS Genet 2013, 9:e1003443.

14. Pollard KS, Hubisz MJ, Rosenbloom KR, Siepel A: Detection of nonneutral substitution rates on mammalian phylogenies. Genome Res 2010, 20:110-121.

15. Cooper GM, Goode DL, Ng SB, Sidow A, Bamshad MJ, Shendure J, Nickerson DA: Single-nucleotide evolutionary constraint scores highlight diseasecausing mutations. Nat Methods 2010, 7:250-251.

16. Tennessen JA, Bigham AW, O'Connor TD, Fu W, Kenny EE, Gravel S, McGee S, Do R, Liu X, Jun G, Kang HM, Jordan D, Leal SM, Gabriel S, Rieder MJ, Abecasis G, Altshuler D, Nickerson DA, Boerwinkle E, Sunyaev S, Bustamante CD, Bamshad MJ, Akey JM, Broad GO, Seattle GO: NHLBI Exome Sequencing Project: Evolution and functional impact of rare coding variation from deep sequencing of human exomes. Science 2012, 337:64-69.

17. Ashkenazy H, Erez E, Martz E, Pupko T, Ben-Tal N: ConSurf 2010: calculating evolutionary conservation in sequence and structure of proteins and nucleic acids. Nucleic Acids Res 2010, 38:W529-533.

18. Wu X, Chi RJ, Baskin JM, Lucast L, Burd CG, De Camilli P, Reinisch KM: Structural insights into assembly and regulation of the plasma membrane phosphatidylinositol 4-kinase complex. Dev Cell 2014, 28:19-29.

19. Kang HJ, Kawasawa Yl, Cheng F, Zhu Y, Xu X, Li M, Sousa AM, Pletikos M, Sedmark G, Guennel T, Shin Y, Johnson MB, Krsnik Z, Mayer S, Fertuzinhos S, Umlauf S, Lisgo SN, Vortmeyer A, Weinberger DR, Mane S, Hyde TM, Huttner A, Reimers M, Kleinman JE, Sestan N: Spatio-temporal transcriptome of the human brain. Nature 2011, 478:483-489.

20. Voineagu I, Wang X, Johnston P, Lowe JK, Tian Y, Horvath S, Mill J, Cantor RM, Blencowe BJ, Geschwind DH: Transcriptomic analysis of autistic brain reveals convergent molecular pathology. Nature 2011, 474:380-384.

21. Parikshak NN, Luo R, Zhang A, Won H, Lowe JK, Chandran V, Horvath S, Geschwind DH: Integrative functional genomic analyses implicate specific molecular pathways and circuits in autism. Cell 2013, 155:1008-1021.

22. Mathieson I, McVean G: Differential confounding of rare and common variants in spatially structured populations. Nat Genet 2012, 44:243-246.

23. Ji W, Foo JN, O'Roak BJ, Zhao H, Larson MG, Simon DB, Newton-Cheh C, State MW, Levy D, Lifton RP: Rare independent mutations in renal salt handling genes contribute to blood pressure variation. Nat Genet 2008, 40:592-599.

24. Gong W, Emanuel BS, Galili N, Kim DH, Roe B, Driscoll DA, Budarf ML: Structural and mutational analysis of a conserved gene (DGSI) from the minimal DiGeorge syndrome critical region. Hum Mol Genet 1997, 6:267-276.

25. Sanders SJ, Ercan-Sencicek AG, Hus V, Luo R, Murtha MT, Moreno-De-Luca D, Chu SH, Moreau MP, Gupta AR, Thomson SA, Mason CE, Bilguvar K, Celestino-Soper PBS, Choi M, Crawford EL, Wright NRD, Dhodapkar RM, DiCola M, DiLullo NM, Fernandez TV, Fielding-Singh V, Fishman DO, Frahm S, Goh GS, Kammela S, Klei L, Lowe JK, Lund SC, McGrew AD, Meyer KA, et al: Multiple recurrent de novo CNVs, including duplications of the 7q11.23 Williams syndrome region, are strongly associated with autism. Neuron 2011, 70:863-885

26. Huang FD, Matthies HJG, Speese SD, Smith MA, Broadie K: Rolling blackout, a newly identified PIP2-DAG pathway lipase required for Drosophila phototransduction. Nat Neurosci 2004, 7:1070-1078.

27. Huang FD, Woodruff E, Mohrmann R, Broadie K: Rolling blackout is required for synaptic vesicle exocytosis. J Neurosci 2006, 26:2369-2379.
28. Huang DW, Sherman BT, Lempicki RA: Systematic and integrative analysis of large gene lists using DAVID bioinformatics resources. Nat Protoc 2009, 4:44-57.

29. Huang DW, Sherman BT, Lempicki RA: Bioinformatics enrichment tools: paths toward the comprehensive functional analysis of large gene lists. Nucleic Acids Res 2009, 37:1-13.

30. Di Paolo G, Moskowitz HS, Gipson K, Wenk MR, Voronov S, Obayashi M, Flavell R, Fitzsimonds RM, Ryan TA, De Camilli P: Impaired Ptdlns (4,5)P2 synthesis in nerve terminals produces defects in synaptic vesicle trafficking. Nature 2004, 431:415-422.

31. He X, Sanders SJ, Liu L, De Rubeis S, Lim ET, Sutcliffe JS, Schellenberg GD, Gibbs RA, Daly MJ, Buxbaum JD, State MW, Devlin B, Roeder K: Integrated model of de novo and inherited genetic variants yields greater power to identify risk genes. PLOS Genet 2013, 9:e1003671.

32. Di Paolo G, De Camilli P: Phosphoinositides in cell regulation and membrane dynamics. Nature 2006, 443:651-657.

33. Hammond GR, Fischer MJ, Anderson KE, Holdich J, Kotecki A, Balla T, Irvine RF: PI4P and PI(4,5)P2 are essential but independent lipid determinants of membrane identity. Science 2012, 337:727-730.

34. Butler MG, Dasouki MJ, Zhou XP, Talebizadeh Z, Brown M, Takahashi TN, Miles JH, Wang $\mathrm{CH}$, Stratton R, Pilarski R: Subset of individuals with autism spectrum disorders and extreme macrocephaly associated with germline PTEN tumour suppressor gene mutations. J Med Genet 2005, 42:318-321.

35. Smalley SL: Autism and tuberous sclerosis. J Autism Dev Disord 1998, 28:407-414.

36. Levitt $P$, Campbell DB: The genetic and neurobiologic compass points toward common signaling dysfunctions in autism spectrum disorders. J Clin Invest 2009, 119:747-754.

37. Judson MC, Eagleson KL, Levitt P: A new synaptic player leading to autism risk: Met receptor tyrosine kinase. J Neurodev Disord 2011, 3:282-292.

38. Marone R, Cmiljanovic V, Giese B, Wymann MP: Targeting phosphoinositide 3-kinase - moving towards therapy. Biochim Biophys Acta 2008, 1784:159-185.

39. Brown JR, Auger KR: Phylogenomics of phosphoinositide lipid kinases: perspectives on the evolution of second messenger signaling and drug discovery. BMC Evol Biol 2011, 11:4.

40. Vadas O, Burke JE, Zhang X, Berndt A, Williams RL: Structural basis for activation and inhibition of class I phosphoinositide 3-kinases. Sci Signal 2011, 4:re2.

41. Ehninger D, Han S, Shilyansky C, Zhou Y, Li W, Kwiatkowski DJ, Ramesh V, Silva AJ: Reversal of learning deficits in a Tsc2+/-mouse model of tuberous sclerosis. Nat Med 2008, 14:843-848.

42. Tsai PT, Hull C, Chu YX, Greene-Colozzi E, Sadowski AR, Leech JM, Steinberg J, Crawley JN, Regehr WG, Sahin M: Autistic-like behavior and cerebellar dysfunction in Purkinje cell Tsc1 mutant mice. Nature 2012, 488:647-651.

doi:10.1186/2040-2392-5-31

Cite this article as: Gupta et al:: Rare deleterious mutations of the gene EFR3A in autism spectrum disorders. Molecular Autism 2014 5:31.

\section{Submit your next manuscript to BioMed Central and take full advantage of:}

- Convenient online submission

- Thorough peer review

- No space constraints or color figure charges

- Immediate publication on acceptance

- Inclusion in PubMed, CAS, Scopus and Google Scholar

- Research which is freely available for redistribution 